\title{
25 Research Suare \\ One-plasmid based CRISPR-Cas9 editing for gene deletion in Lactococcus lactis
}

José Miguel Miquelão Santos

IST: Universidade de Lisboa Instituto Superior Tecnico https://orcid.org/0000-0002-2153-2423

Gabriel António Amaro Monteiro

Técnico Lisboa: Universidade de Lisboa Instituto Superior Tecnico

Duarte Miguel de França Teixeira dos Prazeres

IST: Universidade de Lisboa Instituto Superior Tecnico

Sofia de Oliveira Dias Duarte ( $\nabla$ sofia.duarte@tecnico.ulisboa.pt )

IST: Universidade de Lisboa Instituto Superior Tecnico https://orcid.org/0000-0002-7945-1474

Original article

Keywords: Lactococcus, CRISPR-Cas9, Genome Editing, Cell Factories

Posted Date: March 16th, 2021

DOI: https://doi.org/10.21203/rs.3.rs-306012/v1

License: (c) (1) This work is licensed under a Creative Commons Attribution 4.0 International License.

Read Full License 


\section{Abstract}

Lactococcus lactis strains are promising cell factories and delivery vehicles of plasmid DNA and recombinant protein for therapeutic applications. However, the limited yields of recombinant molecules obtained with these bacteria limits their wide applicability. Genome engineering of this host may solve the problem. However, the current genome editing toolbox available for L. lactis is either too laborious or incapable of large edits, limiting the scope of strain editing experiments. In this work, the basis for a oneplasmid CRISPR-Cas9 based genome editing plasmid was developed and tested. The new plasmid (pTCas9d0) adapted from the pKCcas9d0 plasmid was used to delete $657 \mathrm{bp}$ of the lactococcal nuclease $n$th of L. lactis subsp. lactis LMG19460, with the aim of improving yield and quality of plasmid DNA replicated in this strain. Although deletion mutants were successfully generated, plasmid curing was unsuccessful. Thus, further modifications are required before the plasmid is truly applicable for genome editing experiments. Unexpectedly, the generated deletion mutants generated a roughly $40 \%$ decrease in plasmid yield alongside with a decrease in the quality of produced pDNA.

\section{Introduction}

Over the last decades there has been an increasing interest in the use of Lactococcus lactis as cell factories. Their non-pathogenic nature, coupled to their extensive use in the food industry, make these bacteria safer alternatives to produce active biomolecules than the more widely used Escherichia coli. Although $E$. coli delivers high yields and is easy to use, it produces lipopolysaccharides, requiring costly and timely downstream processes to avoid co-purification with the final product (Wakelin et al. 2006, Wicks et al. 1995, Schneier et al. 2020). The increased safety profile has led to a particular focus on $L$. lactis as both producers and delivery vehicles of biopharmaceuticals, with particular focus on mucosal vaccination with plasmid DNA (pDNA) (Asensi et al. 2013, Wells et al. 1993, Pontes et al. 2003, Pereira et al. 2015, Bermúdez-Humarán et al. 2004). The safe use of L. lactis in this context has been demonstrated with a genome edited human IL-10 producing strain that was tested in clinical trials for the therapy of Crohn's disease (Braat et al 2006).

However, the usually disappointing yields of recombinant molecules produced by L. lactis strains undermine their potential applicability. An improved productivity of high-quality pDNA, for example, would make these bacteria better vectors for mucosal vaccination using DNA vaccines, and would also improve yields in the context of recombinant protein production (Duarte et al. 2021). Genome editing might provide a solution, as demonstrated for a few selected genes. For example, the effects of the $y b d D$ (Morello et al. 2012, Nouaille et al. 2004), clpP and hrtA (Cortes-Perez et al. 2006) genes on recombinant protein production were investigated, with knockout strains exhibiting higher protein yields compared to the wild-type. Larger scale genome editing was also successfully accomplished in L. lactis, as illustrated by the development of NZ9000 genome reduced strains (Liu et al. 2019, Zhu et al. 2017), which exhibited an overall increased robustness and substantially enhanced heterologous protein production. Despite this, current genome editing attempts of L. lactis strains are still limited by the tools available. The reduced genome strains mentioned above, for example, were developed using a Cre-loxP genome editing 
system (Zhu et al. 2015). Although robust and capable of driving knockouts over $20 \mathrm{~kb}$ long, the system is laborious and time-consuming, a feature that prompted the development of improved and easier to use versions (Liu et al. 2019). Faster and more efficient genome editing plasmids exist in L. lactis like the pLRecT (Guo et al. 2019) dual plasmid system, which allows for highly efficient and expedite editing of Lactococcus. However, it is incapable of knockouts roughly $150 \mathrm{bp}$ in size, which limits its use in larger scale genome editing projects. Additionally, both systems employ the use of multiple plasmids and/or ssDNA donors for recombination of the targeted gene, reducing their effectiveness in strains difficult to transform.

CRISPR-Cas 9 based genome editing systems provide quick and effective generation of mutants, with the capacity for large-scale edits, as well. These systems typically function by targeting the Cas 9 protein to a particular gene of interest and inserting a double strand DNA break at the targeted site. This damage leads to the recruitment of the host's homology-directed repair (HDR) mechanisms to the targeted site. Inclusion of the flanking regions of the target in trans, typically in a plasmid, allows the host's HDR mechanisms to substitute the affected region with the repair template. This can be applied to drive deletions, insertions and base edits through careful design of the repair template. Additionally, as chromosomally located DNA breaks tend to be fatal if not repaired, Cas 9 activity also provides selection for cells carrying the desired mutation (Vercoe et al. 2013). As the Cas9 protein requires only the protein itself and a target specific single-guide RNA (sgRNA) to work, CRISPR-Cas9 based genome editing systems can be incorporated into a single plasmid, providing a relatively simple system for a relatively quick genome editing without limitations to the scale of possible edits. Applications employing multiplexed single guide RNA (sgRNA) expression were successfully employed in E. coli (Huang et al. 2019) and Streptomyces coelicor (Huang et al. 2015) to drive deletions of over $80 \mathrm{~kb}$ in a single genome editing event, for example. However, to the best of our knowledge, CRISPR-Cas 9 based genome editing systems for $L$. lactis based on a single plasmid have not be developed so far. Development of such a system would provide a very effective tool for large-scale genome editing experiments in L. lactis, thus contributing to facilitate the engineering of these bacteria.

In this paper we set out to develop a one-plasmid based CRISPR-Cas9 system for the editing of L. lactis. The new plasmid is derived from the pKCcas9dO plasmid developed previously by Huang et al. (Huang et al. 2015) and modified by our group (Duarte 2018) and was used to generate deletion mutants of the lactococcal nuclease $n$th of L. lactis subsp. lactis LMG19460. The 657 bp $n$th gene, which codes for a nuclease with a predicted molecular weight of $24.5 \mathrm{kDa}$, was selected as a target for deletion with the specific aim of obtaining $L$. lactis mutants more appropriate for plasmid replication. Its homologue in $E$. coli codes for Endonuclease III, a repair enzyme with redundant activity (Cunningham et al. 1985, Saito et al. 1995). We reasoned that by deleting $n$ th, the yields and quality of plasmid DNA replicated in L. lactis could potentially be improved by limiting non-specific digestion.

\section{Methods}

\section{Bacterial Strains, Plasmids and Growth Conditions}


E. coli DH5a cells were used as the host for all plasmids during plasmid construction. L. lactis subsp. lactis IL1403 (Chopin et al. 1984) was used as the source of the $\mathrm{P}_{\mathrm{XylT}}$ promoter and L. lactis subsp. lactis LMG19460 was used as the final recipient of the constructed plasmids and the target of the nth gene deletion. All strains used in this work are listed in Table 1.

Table 1 - Bacterial strains used in this work, alongside their relevant characteristics and source.

\begin{tabular}{|c|c|c|}
\hline $\begin{array}{l}\text { Species } \\
\text { and Strain }\end{array}$ & Relevant Genotype & Source \\
\hline $\begin{array}{l}\text { E. coli } \\
\text { DH5a }\end{array}$ & $\begin{array}{l}\mathrm{F}-\text { Ф80lacZ } \Delta \mathrm{M} 15 \Delta(\text { IacZYA-argF) U169 recA1 endA1 hsdR17 (rK-, } \\
\mathrm{mK}+\text { ) phoA supE44 } \lambda-\text { thi-1 gyrA96 relA1 }\end{array}$ & Invitrogen \\
\hline $\begin{array}{l}\text { L. lactis } \\
\text { LMG19460 }\end{array}$ & Plasmid-free wild type strain & $\begin{array}{l}\text { LMG/BCCM } \\
\text { (Belgium) }\end{array}$ \\
\hline \multirow[t]{2}{*}{$\begin{array}{l}\text { L. lactis } \\
\text { IL1403 }\end{array}$} & Plasmid-free wild type strain & $\begin{array}{l}\text { Chopin et al. } \\
1984\end{array}$ \\
\hline & & $\begin{array}{l}\text { INRAE, } \\
\text { France }\end{array}$ \\
\hline
\end{tabular}

The pTRKH3 plasmid has been described before (O'Sullivan et al. 1993) and was used as the new vector backbone for the constructed plasmids while the pKCcas9dO_nth_apra (Duarte 2018) plasmid was used as the source of the Cas9 gene sequence and the $n$th gene specific single-guide RNA and repair template. The pKCcas9dO_nth_apra (Duarte 2018) plasmid is derived from the pKCcas9dO plasmid (Huang et al. 2015) and contains a S. coelicor optimized Cas9 (ScoCas9) gene sequence under the control of the thiostrepton inducible $\mathrm{P}_{\mathrm{TipA}}$ promoter, a constitutive promoter named J23119, downstream of which is an nth gene specific sgRNA and the minimal replicon of the thermosensitive plasmid pSG5 (Muth et al. 1988, Muth et al. 1989)). Additionally, the plasmid also contains an $n$th specific repair template, or homologyarms (HAs). This constitutes two regions, 1,028 and $955 \mathrm{bp}$ in size, that share homology to the flanking regions of the $n$th gene sequence in the L. lactis LMG19460 genome. All plasmids used in this work, alongside their relevant characteristics, are listed in Table 2.

Table 2 - All plasmids used in this work, alongside their relevant characteristics and source. 


\begin{tabular}{|c|c|c|}
\hline Name & Relevant Characteristics & Source \\
\hline \multirow[t]{2}{*}{ pTRKH3 } & p15A ori, $\mathrm{pAM} \beta 1$ ori, tet $R$, ery $R$ & $\begin{array}{l}\text { O'Sullivan et al. } \\
1993\end{array}$ \\
\hline & & $\begin{array}{l}\text { LMBP/BCCM } \\
\text { (LMBP 4462) } \\
\text { (Belgium) }\end{array}$ \\
\hline \multirow[t]{2}{*}{ pKCcas9dO_nth_apra } & pSG5 ori, apmR & Duarte 2018 \\
\hline & $n t h$ specific sgRNA and repair template & \\
\hline \multirow[t]{2}{*}{ pTXylT } & p15A ori, $\mathrm{pAM} \beta 1$ ori, ery $R$ & This work \\
\hline & $\begin{array}{l}\text { PxylT } \\
\text { p15A ori }\end{array}$ & \\
\hline \multirow[t]{2}{*}{ pTScoCas9 } & p15A ori, $\mathrm{pAM} \beta 1$ ori, ery $R$ & This work \\
\hline & $\begin{array}{l}\text { Cas9 sequence from pKCcas9dO_nth_apra inserted } \\
\text { downstream of } P_{X y l T} \text { promoter }\end{array}$ & \\
\hline \multirow[t]{2}{*}{ pTCas9dO } & p15A ori, $\mathrm{pAM} \beta 1$ ori, ery $R$ & This work \\
\hline & $\begin{array}{l}\text { nth specific sgRNA and repair template from } \\
\text { pKCcas9dO_nth_apra inserted upstream of } \mathrm{P}_{\text {XylT }} \\
\text { promoter }\end{array}$ & \\
\hline
\end{tabular}

E coli DH5a cells were typically grown in Luria-Bertani (LB) broth (NZYTech) at $37^{\circ} \mathrm{C}, 250 \mathrm{rpm}$. On solid media, cells were plated onto LB agar (NZYTech) and incubated at $37^{\circ} \mathrm{C}$. Incubation was done at $30^{\circ} \mathrm{C}$ when cells were transformed with thermosensitive plasmids (pKCcas9dO_nth_apra). When needed, Erythromycin or Apramycin were added at $500 \mu \mathrm{g} / \mathrm{mL}$.

L. lactis subsp. lactis LMG19460 and IL1403 cells were grown in M-17 medium (Sigma-Aldrich) supplemented with either $20 \mathrm{~g} / \mathrm{L}$ of $D(+)$-glucose (GM-17) or $20 \mathrm{~g} / \mathrm{L}$ of $D(+)$-xylose $(\mathrm{XM}-17)$, at $30^{\circ} \mathrm{C}, 100$ rpm. On solid media, cells were incubated on either modified Lactic Agar (Elliker et al. 1956) $(5 \mathrm{~g} / \mathrm{L}$ lactose, $20 \mathrm{~g} / \mathrm{L}$ tryptone, $5 \mathrm{~g} / \mathrm{L}$ yeast extract, $4 \mathrm{~g} / \mathrm{L}$ sodium chloride, $1.5 \mathrm{~g} / \mathrm{L}$ sodium acetate, $0.5 \mathrm{~g} / \mathrm{L} \mathrm{L}$ ascorbic acid and $15 \mathrm{~g} / \mathrm{L}$ agar) or SR medium (Holo et al. 1989) at $30^{\circ} \mathrm{C}$. Erythromycin was added at 5 $\mathrm{ng} / \mu \mathrm{L}$, when needed.

\section{General Cloning Procedures}

Plasmid DNA was obtained from E. coli and L. lactis cells using the High Pure Plasmid Isolation Kit (Roche) and the Nucleospin Plasmid Purification Kit (Macherey-Nagel), respectively. L. lactis strains' genomic DNA (gDNA) was obtained using the Wizard Genomic DNA Purification Kit (Promega). The NZYGelpure Kit (NZYTech) was used for extraction of DNA fragments from agarose gels and clean-up of PCR and restriction reaction products. Sanger sequencing was done through Stabvida (Portugal). 
All amplification reactions for cloning purposes were done using the KOD Hot Start DNA polymerase (Novagen), with the exception of the amplification reaction using the pTRKH3:pXylT:ScoCas9_F/R primer pair, which was done using the Supreme NZYLong Colourless Master Mix (NZYTech) polymerase. Amplification reactions for confirmation of the $n$th gene knockout were done using the NovaTaq Hot Start Master Mix (Novagen) enzyme and nth_conf_F/R primer pair. All primers used are listed in Table 3.

\section{Table 3 - Primers used in this work.}

\begin{tabular}{|ll|}
\hline Primer Name & Sequence \\
\hline pTRKH3_F & AGTCTAGAATCGATACGATTTTG \\
\hline pTRKH3_R & TTGAGATCGTTTTGGTCTG \\
\hline pXylT_F & GCAGACCAAAACGATCTCAACATAAAAACTACTCTTTACATAAGAG \\
\hline pXylT_R & AATCGTATCGATTCTAGACTTTTTTATTTGCAAGTCTTCTTG \\
\hline pTRKH3:pXyIT_Res_F & CGCTGTACAAGTCTAGAATCGATACGATTTTG \\
\hline pTRKH3:pXyIT_Res_R & CATGCCATGGTTTTTATTTGCAAGTCTTCTTGC \\
\hline ScoCas9_Res_F & CATGCCATGGAGAAGGGAGCGGACATATGGACAAG \\
\hline ScoCas9_Res_R & CGCTGTACATCAGTCGCCGCCCAGCTG \\
\hline pTRKH3:pXyIT:ScoCas9_F & CATAAAAACTACTCTTTACATAAGAGTAGTTTTTAG \\
\hline pTRKH3:pXyIT:ScoCas9_R & TTGAGATCGTTTTGGTCTG \\
\hline sgRNA+HAs_F & $\underline{\text { GCAGACCAAAACGATCTCAACAGCTCGCGCCAAGTCCA }}$ \\
\hline sgRNAs+HAs_R & $\underline{\text { TGTAAAGAGTAGTTTTTATGTTGACAGCTAGCTCAGTCCTAGG }}$ \\
\hline nth_conf_F & CAAGGGCAAGTCAAATATAC \\
\hline nth_conf_R & GTATGGCTGGTATAGACAGCA \\
\hline
\end{tabular}

Underlined bases represent the region of the primer which anneals exclusively to its complementary Gibson Assembly pair. Bases in bold represent the added restriction sites for the BsrGl enzyme while the bases in bold and italics represent the added restriction sites for the Ncol enzyme.

Restriction endonucleases used were Dpnl (Thermo Fisher Scientific), Ncol (Thermo Fisher Scientific), BsrGI (Thermo Fisher Scientific), BamHI (Promega) and HindlII (Promega) enzymes. All PCR products intended for ligation were incubated for 3 hours with Dpnl enzyme. Ligation reactions were mostly done through the Gibson Assembly ${ }^{\circledR}$ Master Mix (New England Biolabs), except for the construction of the pTScoCas9 intermediary plasmid which was done using a T4 DNA Ligase enzyme (Promega). Ligation products were transformed into competent $E$. coli DH5a cells and plated onto LB agar with appropriate antibiotic. 
All kits and enzymes were used in accordance with manufacturer's guidelines.

Chemically competent $E$. coli DH5a cells were prepared and transformed as previously described by Chung et al. (1989), with the following modifications: competent cells were prepared from cultures at an optical density at $600 \mathrm{~nm}(0 \mathrm{D} 600 \mathrm{~nm})$ of 0.95 , transformation was done with $100 \mathrm{ng}$ of plasmid per transformation and cells were given a $42^{\circ} \mathrm{C}$ heat shock, in a water bath, for 1 minute following the $4^{\circ} \mathrm{C}$ incubation period. Transformants were selected by plating in antibiotic supplemented LB agar.

L. lactis LMG19460 cells were made competent and transformed as previously described by Holo et al. (1989) with slight modifications. Electroporation was done using a Gene Pulser (Bio-Rad Laboratories) with a Pulse Controller (Bio-Rad Laboratories) and cells were given 2 to 5 shocks of $10.0 \mathrm{kV} / \mathrm{cm}, 400 \Omega$ and $25 \mu \mathrm{F}$. Transformants were selected by incubation in SR medium supplemented with erythromycin.

\section{Induction of Cas9 Expression}

Induction of the pTCas9dO plasmid was done as described previously by Miyoshi et al. (2004) with slight modifications. Transformed L. lactis LMG19460 cells were grown overnight in GM-17 until confluency. These cultures were then pelleted and washed twice with fresh M-17 medium, to remove most traces of glucose, before being inoculated into $X M-17$, such that the starting $\mathrm{OD}_{600 \mathrm{~nm}}$ of these new cultures was 0.1. After overnight growth, appropriate dilutions of the cultures were prepared and plated onto erythromycin supplemented Lactic Agar plates to isolate possible mutants. The efficiency of the plasmid for deletion of the $n$th gene was calculated as described previously (Song et al. 2017).

\section{pTCas9d0 Plasmid Curing}

Curing attempts of the pTCas9dO plasmid were done through extended incubation at higher temperatures and protoplast regeneration. For plasmid curing by extended incubation, pTCas9dO transformed $\Delta$ th LMG19460 cells were inoculated into erythromycin free GM-17 medium and incubated at $32^{\circ} \mathrm{C}, 37^{\circ} \mathrm{C}$ and $40^{\circ} \mathrm{C}$ for up to 120 hours, in the same culture. Appropriate dilutions were then plated onto SR medium for colony counting at 96 and 120 hours of incubation.

Longer incubation times, in both liquid and solid media, were also attempted. pTCas9d0 transformed $\Delta n$th LMG19460 cells were grown in erythromycin free GM-17 medium for a total of 26 days, with cells being transferred to fresh GM-17 medium every day to ensure the culture's viability. Appropriate dilutions of the cultures were plated onto erythromycin free Lactic Agar plates before every transfer. On solid medium, pTCas9dO carrying deletion mutants were plated onto erythromycin free Lactic Agar plates. Every two days, of the obtained colonies, 6 were streaked onto new plates while another 50 were tested for loss of plasmid.

Curing attempted through protoplast regeneration was done as previously described by Mastrigt et al. (2018). 
Following all protocols, screening for loss of plasmid from the obtained colonies was done through replica plating onto Lactic Acid Agar, with and without erythromycin.

\section{Quantification of pDNA yield and pDNA quality analysis}

pTCas9dO transformed wild-type and $\Delta$ th L. lactis LMG19460 cells were grown up to 48 hours in M-17 medium supplemented with $0.5 \%$ glucose. Samples were taken at late-exponential phase $\left(\mathrm{OD}_{600 \mathrm{~nm}}\right.$ of 1.6) and at 24 hours following start of incubation. The cell count was standardized for each sample by diluting enough culture to obtain $5 \mathrm{~mL}$ samples with an $\mathrm{OD}_{600 \mathrm{~nm}}$ of roughly 1.6 . These samples were pelleted and used for pDNA purification.

pDNA yield was assessed spectrophotometrically using a NanoDrop One (Thermo Fisher Scientific) instrument while pDNA quality was assessed by comparing the relative intensity of the plasmid's isoforms in a $0.8 \%(\mathrm{w} / \mathrm{V})$ agarose gel, loaded with $2 \mu \mathrm{g}$ of pDNA.

\section{Statistical analysis}

Statistically significant differences in pDNA yield were determined using an unpaired, two-tailed t-test, performed using GraphPad Prism version 9.0.2 for Windows, GraphPad Software, San Diego, California USA, www.graphpad.com.

\section{Results}

\section{Plasmids in silico design}

In previous work we used the pKCcas9dO (Huang et al. 2015) plasmid to generate pKCcas9dO_nth_apra, a plasmid that carries an $n$th gene specific sgRNA and repair template (Duarte 2018). However, this plasmid was ineffective to knockout L. lactis, due to the inability of the thiostrepton-inducible promoter to successfully induce Cas9 expression. Additionally, the $a p m R$ resistance marker from pKCcas9d0 plasmid is inappropriate for L. lactis LMG19460 as this strain is highly resistant to apramycin. We thus designed the new plasmid pTCas9dO using pKCcas9d0_nth_apra as a starting point by replacing the promoter for Cas9 expression for the xylose inducible and glucose repressible $\mathrm{P}_{\text {XylT }}$ promoter (Miyoshi et al. 2004), and by introducing the widely used PTRKH3 plasmid as a new backbone. Key features of this new backbone include an effective erythromycin selection marker, a medium copy number Gram-negative origin of replication ( $15 \mathrm{~A}$ ), and a high copy number Gram-positive PAM 11 origin of replication. The tetracyclin resistance marker (tetR) was also excluded to decrease the size of the final construct, since the erythromycin resistance marker (ery $R$ ) is sufficient for effective selection in both $E$. coli and L. lactis. The $n$th gene specific sgRNA and repair template, which target a $657 \mathrm{bp}$ region of $n$th for deletion, were obtained from the pKCcas9dO_nth_apra plasmid. The desired components of each plasmid were amplified using primers designed for Gibson Assembly, except for the construction of the pTScoCas9 plasmid, which was amplified with primers containing added restriction sites for the $\mathrm{Ncol}$ and $\mathrm{BsrGI}$ enzymes and ligated through a T4 DNA ligase reaction. A schematic of the steps involved in the 
construction of the plasmid can be seen in Supplemental Figure 1, while the final construct is shown in Figure 1.

Figure 1 - Schematics of the final constructed plasmids, pTCas9d0. Relevant restriction sites, for the BsrGI and Ncol (plasmid construction) and BamHI and HindIII (screening of transformants) enzymes were included. p15A - Gram-negative, medium copy number origin of replication; pAMß1 - Grampositive, high copy number origin of replication; eryR - Erythromycin selection marker; $\mathrm{pXyIT}$ Promoter Xylose inducible $\mathrm{P}_{\text {XylT }}$ promoter; J23119 - Constitutive promoter; ScoCas9 - S. coelicor codon-optimized Cas9 gene sequence; $n$th sgRNA - nth gene specific sgRNA; $n$th HA 1-2 - nth gene specific homologyarms or repair template, constituting the 1,028 (nth HA1) and 955 bp (nth HA2) fragments that share homology with the flanking regions of the $n$th gene; Image prepared using SnapGene software (from Insightful Science; available at snapgene.com).

\section{pTCas9d0 construction}

Firstly, the $\mathrm{P}_{\text {XylT }}$ promoter was inserted into the new $\mathrm{pTRKH3}$ vector backbone. The promoter was amplified from L. lactis IL1403 gDNA using the pXyIT_F/R primer pair, while the vector backbone was amplified from the pTRKH3 plasmid using the pTRKH3_F/R primer pair. This amplification excluded the ribosomal binding site (RBS) of the $\mathrm{P}_{\mathrm{XylT}}$ promoter, as this region has an unfavourable $\mathrm{G}+\mathrm{C}$ mol\% content for effective amplification and Gibson Assembly ligation. The desired amplification products were purified from an agarose gel and ligated using Gibson assembly to obtain the pTXyIT intermediary plasmid. Cells transformed with the ligation products were used for pDNA purification and the correct insert was confirmed by amplification using the pXyIT_F/R primer pair (Figure 2a). The correct plasmid was successfully obtained and confirmed through sequencing.

The pTXyIT plasmid was then linearized through amplification using the pTRKH3:pXyIT_F/R primer pair while the Cas9 gene sequence, with the RBS of the $\mathrm{P}_{\mathrm{TipA}}$ promoter immediately upstream, was also obtained through amplification of the pKCcas9d0_nth_apra plasmid. This primer pair was designed to contain additional restriction sites for the BsrGI and Ncol enzymes, which allowed ligation of the Cas9 gene sequence immediately downstream of the $P_{\text {XylT }}$ promoter using a T4 DNA ligase, resulting in the pTScoCas 9 intermediary plasmid. Screening of cells transformed with the resulting ligation products was done through a restriction profile using the BamHI and Hindlll enzymes (Figure 2a). Of the screened colonies, one (clone number 4 in Figure 2a) exhibited the expected pattern of 6,639 and 3,882 bp.

Finally, the sgRNA sequence for the $n$th gene, alongside the $n$th specific repair template, were amplified from the pKCcas9dO_nth_apra plasmid using the sgRNA+HAs_F/R primer pair. This fragment was inserted into the pTScoCas9 plasmid, which was previously linearized through amplification using the pTRKH3:pXyIT:ScoCas9_F/R primer pair. Ligation was done through Gibson Assembly, resulting in the pTCas9dO plasmid. The resulting transformants were screened by a restriction profile using the BsrGI enzyme (Figure 2b). One clone (clone 1 of Figure 2b) exhibited the expected pattern of 7,471 and 5,172 bp. The plasmid sequence was further verified through sequencing. 
Figure $2-1 \%(w / V)$ agarose gels of every screening step of pTCas9d0 construction. All molecular markers used were NZYDNA Ladder III (NZYTech). (a) Restriction profile of transformants obtained from pTScoCas9 ligation reaction using the BamHI and Hindlll enzymes. Non-restricted samples $(\mathrm{N})$ were ran alongside their restriction products (R). A positive control, composed of a previously obtained pTScoCas 9 solution unusable due to mutation (C+), was included. One clone (4) exhibited the correct pattern of 6,639 and 3,882 bp. (b) Restriction profile of transformants obtained from pTCas9dO ligation reaction using the BsrGI enzyme. A negative control (C-) was included using the pTScoCas9 plasmid. Non-restricted samples $(\mathrm{N})$ were ran alongside their restriction products $(\mathrm{R})$, and one clone (2) exhibited the correct restriction pattern of 7,471 and 5,172 bp.

\section{The nth gene knockout was successfully obtained with the pTCas9dO plasmid}

The pTCas9dO plasmid was transformed into L. lactis LMG19460 cells and the resulting transformants were induced by incubation in XM-17 medium. The induced cultures exhibited a noticeable decrease of cell density 3 hours after induction $\left(\mathrm{OD}_{600 \mathrm{~nm}}\right.$ of $0.126 \pm 0.006$ to $0.083 \pm 0.006$ in 2 hours), most likely because of Cas9-induced cell death. The cultures maintained a lower cell density $\left(\mathrm{OD}_{600 \mathrm{~nm}}\right.$ of roughly 0.09) for over 24 hours following induction. Culture samples were plated after the cells were induced overnight. The resulting colonies were screened for the deletion by amplification of gDNA purified from individual colonies using the nth_conf_F/R primer pair (Figure 3).

Of the 23 colonies tested after pTCas9dO induction, 18 showed an observable amplicon. Among these, 8 exhibited the amplicon characteristic of the $n$th gene knockout $(2,133 \mathrm{bp})$, while another 6 exhibited the wild-type amplicon $(2,790 \mathrm{bp})$. The remaining 4 colonies appeared mixed as they exhibited both amplicons. An efficiency of $66.7 \%$ was calculated for the deletion of the $657 \mathrm{bp} n$th gene sequence using the pTCas9dO plasmid.

\section{General Curing Procedures Were Incapable of Curing the pTCas9dO}

Following knockout of the $n$th gene, attempts were the made to cure the plasmid pTCas $9 \mathrm{dO}$ from the successful clones. Deletion mutants carrying the pTCas9dO plasmid were subjected to extended incubations at $32^{\circ} \mathrm{C}, 37^{\circ} \mathrm{C}$ and $40^{\circ} \mathrm{C}$ in erythromycin free GM-17 medium for a minimum of 96 hours and up to 120 hours. Samples of these cultures were taken at both the 96 and 120 hour-mark for all temperatures and plated onto erythromycin free SR medium. Colonies were only successfully obtained for the plates grown at $32^{\circ} \mathrm{C}$, with only one colony obtained after 120 hours of incubation. Plates obtained from cultures grown at $37^{\circ} \mathrm{C}$ and $40^{\circ} \mathrm{C}$ did not exhibit any growth when plated undiluted onto SR medium plates. Of the plates obtained from the cultures incubated at $32^{\circ} \mathrm{C}$ for 96 hours, a total of 150 colonies were patch plated to check for loss of plasmid, alongside the one colony obtained from the cultures incubated at $32^{\circ} \mathrm{C}$ for 120 hours. Unfortunately, all tested colonies were capable of growing in erythromycin supplemented medium, implying the pTCas9dO was still present.

Curing was also attempted by successively incubating the cells in erythromycin free GM-17. pTCas9dO bearing deletion mutant cells were incubated in GM-17 medium, with the culture being diluted into fresh 
medium daily. Before each transfer, appropriate dilutions of the culture were plated onto erythromycin free Lactic Agar medium. Fifty colonies for each of the plates were then patch plated onto Lactic Agar plates, with and without erythromycin, to check for loss of plasmid. A similar strategy was attempted with solid medium, wherein six colonies of pTCas9dO carrying deletion mutant cells were successively streaked onto erythromycin free Lactic Agar plates. Every two days, 50 colonies were patch plated onto Lactic Agar plates, with and without erythromycin, to check for loss of plasmid, while another 6 colonies were chosen and transferred to new Lactic Agar plates. The 1,300 screened colonies obtained from incubation in GM-17 medium, and the 750 screened colonies obtained from incubation in Lactic Agar were all capable of growing in erythromycin supplemented medium.

A final attempt at pTCas9dO curing was done through protoplast regeneration. Plasmid bearing cells were washed twice with $30 \mathrm{mM}$ Tris-HCl buffer, before being incubated at $37^{\circ} \mathrm{C}$, for 1 or 3 hours, in lysis buffer. The resulting cell suspension was then plated onto SR medium to allow protoplast regeneration for up to 72 hours. The resulting colonies were then patch plated onto erythromycin supplemented and erythromycin free Lactic Agar. In total, 150 colonies were tested for cells incubated in lysis buffer for 1 hour and another 50 were tested for cells incubated for 3 hours. None of the tested colonies exhibited loss of erythromycin resistance.

\section{Deletion of the $n$th gene results in a decrease of total pDNA produced with no noticeable differences in plasmid quality}

Although we were unable to cure pTCas $9 \mathrm{dO}$ from the $n$th deletion mutants, we proceeded to analyse the effects of the deletion on pDNA productivity and quality by examining the replication of the pTCas9dO. To do this, wild-type and $\Delta n$th mutant LMG19460 cells carrying the plasmid were grown in GM-17 medium until late-exponential phase $\left(\mathrm{OD}_{600 \mathrm{~nm}}\right.$ of roughly 1.6$)$ and stationary phase (24 hours of growth) wherein samples of each culture were taken and used to purify pDNA. Monitoring of cell growth throughout this incubation (Supplemental Figure 2) showed that there was a slight increase in both the specific growth rate and final biomass of the $\Delta n t h$ mutants when compared to the wild-type cells. Specifically, the deletion mutants reached a specific growth rate of roughly $0.69 \pm 0.06$ and an average final $\mathrm{OD}_{600 \mathrm{~nm}}$ of 2.82, whereas the wild-type cells reaching a specific growth rate of $0.56 \pm 0.32$ and an $\mathrm{OD}_{600 \mathrm{~nm}}$ of 2.45. pDNA isolated from both the wild-type and deletion mutants was quantified (Figure 4a). At the late exponential phase, there was a significant decrease in pDNA yield (in $\mu \mathrm{g} / \mathrm{L}$ of culture) for the deletion mutants of roughly $40 \%$ when compared to the wild-type cells, while no significant difference was observed for the stationary phase. Furthermore, the relative intensity of the bands for the different isoforms of the plasmids appeared slightly fainter for the $n$th deletion mutants, regardless of growth phase (Figure 4b), implying an overall decrease in pDNA quality and quantity for the $n$th deletion mutants. Several unexpected bands appeared most notably the heavier of which is probably genomic DNA and a $4 \mathrm{~kb}$ apparent size fragment in the wild-type cells. As the strain used is a plasmid-free strain, it is possible that the observed extra bands are the result of undesired recombination events with the pTCas9dO plasmid. This should be an effect of the $n$th deletion as L. lactis LMG19460 cells transformed with other plasmids (pTRKH3) do not exhibit this behaviour. 
Figure 4 - Results for the effect of the $n$th deletion on plasmid yield and quality. (a) Graph showing the average calculated plasmid yield ( $\mu \mathrm{g}$ per litre of culture), from duplicate cultures, for the wild-type and deletion mutant ( $\Delta n t h)$ L. lactis LMG19460 cells, at late exponential $\left(\mathrm{OD}_{600 \mathrm{~nm}}\right.$ of 1.6$)$ and stationary (24 hours) phases of growth. The left graph shows results for the late-exponential phase while the right one shows results for the stationary phase. Asterisks represent statistically significant differences (* - P-value $\leq 0.05$, $\star *$ - P-value $\leq 0.01$ ) as determined by an unpaired two-tailed t-test. (b) $0.8 \%(\mathrm{w} / \mathrm{V})$ agarose gel of $2,000 \mathrm{ng}$ of the pTCas9d0 plasmid obtained from duplicate cultures of wild-type and $\Delta$ th cells, at lateexponential $\left(\mathrm{OD}_{600 \mathrm{~nm}}\right.$ of 1.6$)$. (c) $0.8 \%(\mathrm{w} / \mathrm{V})$ agarose gel of $2,000 \mathrm{ng}$ of the pTCas $9 \mathrm{dO}$ plasmid obtained from duplicate cultures of wild-type and $\Delta n t h$ cells and stationary (24 hours) phases of growth. The pTCas9dO's supercoiled (SC), open-circular (OC) and linear (L) isoforms are marked in each image. The unidentified bands are marked with an asterisk $\left(^{\star}\right)$. The molecular marker for every gel is the NZYDNA Ladder III (NZYTech).

\section{Discussion}

In this work we adapted a one-plasmid CRISPR-Cas9 based genome editing system, previously developed for use in S. coelicor (Huang et al. 2015), for use in L. lactis LMG19460. To the best of the authors knowledge, this is the first time such a system has been applied in a single plasmid in L. lactis. Key features of the resulting $12 \mathrm{kbp}$ pTCas $9 \mathrm{dO}$ plasmid include the erythromycin resistance gene, the pAM $\beta 1$ origin and a CRISPR-Cas9 system. As a proof of concept, we designed the pTCas9d0 plasmid with and RNA guide directed towards creation of a knockout mutant for the lactococcal nuclease $n$ th. Successful mutant strains are expected to result in improved replication of pDNA both in terms of yield and quality.

The 657 bp $n$th gene knockout in L. lactis LMG19460 was successfully obtained with the pTCas9dO plasmid, with $66.7 \%$ of tested clones registering a gene editing event and $44 \%$ of clones being pure deletion mutants. While these efficiencies were satisfactory, other genome editing systems used in Lactic Acid Bacteria have been shown to be capable of higher efficiencies. The Cas9 nickase based plasmid pLCNICK developed for Lactobacillus casei, for example, yielded deletions of similar size with efficiencies of up to $65 \%$ before subculturing and $100 \%$ after sub-culturing (Song et al. 2017), while the previously mentioned pLRecT plasmid reached genome editing efficiencies of over 75\% (Guo et al. 2019). Improving the expression of the Cas9 protein through codon-optimization or the substitution of the $\mathrm{P}_{\text {XylT }}$ promoter with stronger ones like the widely used NICE system (Mierau et al. 2015) could lead to similarly high efficiencies in our plasmid. The pTCas9dO plasmid's relative simplicity allows for hassle-free generation of mutants, requiring only transformation of the plasmid, followed by induction of Cas 9 expression. However, the greatest advantage of our system is the use of a fully-functioning Cas9 protein to drive the knockout of the gene. This should allow the plasmid to drive large-scale deletions if two sgRNAs are employed, with each targeting one of the extremes of the desired region (Huang et al. 2015, Huang et al. 2019), allowing for similar deletion sizes as those demonstrated by Cre-loxP systems (Liu et al. 2019, Zhu et al. 2017), but faster, without employing multiple plasmids or requiring laborious screening of generated mutants. The capacity for multiplexed sgRNA's in our plasmid, however, still needs to be verified. 
While the capacity for genome editing of the plasmid was demonstrated, its curing was unsuccessful. This likely due to the high stability and copy number of the pAM $\beta 1$ origin used (O'Sullivan et al. 1993) (45-80 copies). Nevertheless, curing should be possible given that a similar plasmid containing the same origin was removed by successive streaking in solid medium without antibiotic supplementation (Guo et al. 2019). Repeating the curing procedures in unbuffered medium could provide a solution as the rapid acidification of the medium has been shown to increase plasmid instability (Sinha 1989), but this might make plasmid curing too time-consuming and unreliable. The use of Gram-positive thermosenstive replicons would be more likely to succeed. With this in mind, we are currently modifying the pTCas9dO plasmid by substituting both of its origins of replication with the thermosensitive version of the origin of replication of the lactococcal plasmid pWV01 (Maguin et al. 1992). This origin has been used in plasmids in L. lactis strains for insertional mutagenesis (Maguin et al. 1996) and is readily curable in just 8 hours, which would facilitate the application of the pTCas9dO plasmid for iterative generation of mutants.

Although difficult to ascertain due to the presence of unexpected DNA in the generated deletion mutants, the effects of the $n$th deletion on plasmid yield and quality were also verified, with the generated mutants presenting roughly half the yield of pDNA when compared to those of the wild-type cells. Plasmid quality also appeared to be slightly lower for the deletion mutants as the supercoiled isoform of the plasmids appeared less intense when loaded onto an agarose gel when compared to the wild-type cells, although the difference was very slight. The $n$th gene is identified as an Endonuclease III homologue (through sequence homology), which is a nuclease responsible for excision of bases damaged by oxidation or radiation in E. coli (Cunningham et al. 1985, Matsumoto et al. 2001, Saito et al. 1997). It is possible that the decreased yield and quality of pDNA replicated in the $n$th deletion mutants is due to the accumulation of unrepaired bases. These results are interesting as there was a slight but noticeable increase in both specific growth rate and final biomass of the $n$th deletion mutants when compared to the wild-type cells. It would be expected that the accumulation of DNA damage due to lack of repair would lead to cell death or loss of growth. It is possible that the increase in growth is due to the lower yield of pDNA caused by the mutation, leading to a smaller metabolic burden on the cells. The likely increase in mutations caused by the lack of a DNA repair enzyme could have also led to unexpected mutations, and this behaviour could be due to a random mutation. With plasmid cured deletion mutants the results could be more accurately assessed. Additionally, a widely used plasmid, such as the pTRKH3 plasmid, could be used to assess the pDNA yield and quality more accurately and ensuring more comparable results between different mutants. This would also allow for more accurate estimation of plasmid yield and quality as it would avoid the presence of the undesired fragments observed in the agarose gels. Other methods should also be used to provide more accurate results. Real-time quantitative PCR targeting the pTRKH3 plasmid could be used to assess plasmid yield (Duarte et al. 2019), while fluorescence (Levy et al. 2000) or chromatography (Abdulrahman et al. 2018) based methods could allow for more accurate estimation of the relative abundance of the produced plasmids isoforms.

In closing, we demonstrated that a one-plasmid, CRISPR-Cas9 genome editing plasmid, is viable in $L$. lactis and although the plasmid is still incomplete, it provides a basis for a quick and robust genome editing system for these bacteria. 


\section{Abbreviations}

gDNA - genomic DNA

GM-17 - M-17 medium supplemented with $20 \mathrm{~g} / \mathrm{L}$ glucose

HAs - homology-arms

HDR - Homology-directed repair

$\mathrm{OD}_{600 \mathrm{~nm}}-$ Optical density at $600 \mathrm{~nm}$

pDNA - plasmid DNA

RBS - ribosomal binding site

ScoCas 9 - S. coelicor codon-optimized Cas 9

sgRNA - single-guide RNA

XM-17 - M-17 medium supplemented with $20 \mathrm{~g} / \mathrm{L}$ xylose

\section{Declarations}

\section{Ethics approval and consent to participate}

Not Applicable

\section{Consent for publication}

Not Applicable

\section{Availability of data and materials}

The datasets used and/or analysed during the current study are available from the corresponding author on reasonable request.

\section{Competing Interests}

The authors declare that they have no conflict of interest.

\section{Funding}

This work was made possible by funding received by iBB - Institute for Bioengineering and Biosciences from FCT - Portuguese Foundation for Science and Technology (UID/BIO/04565/2019) and from 
Programa Operacional Regional de Lisboa 2020 (Project N. 007317). The authors acknowledge Fundação para a Ciência e a Tecnologia (FCT, Portugal) the project grant (PTDC/BTM-SAL/28624/2017).

\section{Authors Contributions}

José Santos wrote the manuscript and conducted and designed experiments, Gabriel Monteiro wrote the manuscript and designed experiments, Miguel Prazeres wrote the manuscript and designed experiments and Sofia Duarte wrote the manuscript and conducted and designed experiments.

\section{Acknowledgements}

We thank Marie-Pierre Chapot-Chartier (INRAE) for kindly supplying the Lactococcus lactis IL1403 strain.

\section{References}

Abdulrahman, A, Ghanem, A (2018) Recent advances in chromatographic purification of plasmid DNA for gene therapy and DNA vaccines: a review. Analytica Chimica Acta 1025:41-57.

doi:10.1016/j.aca.2018.04.001

Asensi, GF, de Sales, NFF, Dutra, FF, Feijó, DF, Bozza, MT, Ulrich, RG, Miyoshi, A, de Morais, K, Azevedo, VAC, Silva, JT, Le Loir, Y, Paschoalin, VMF (2013) Oral immunization with Lactococcus lactis secreting attenuated recombinant staphylococcal enterotoxin $B$ induces a protective immune response in a murine model. Microbial Cell Factories 12:32. doi:10.1186/1475-2859-12-32

Bermúdez-Humarán, LG, Cortes-Perez, NG, Le Loir, Y, Alcocer-González, JM, Tamez-Guerra, RS, de OcaLuna, RM, Langella, P (2004) An inducible surface presentation system improves cellular immunity against human papillomavirus type $16 \mathrm{E} 7$ antigen in mice after nasal administration with recombinant lactococci. Journal of Medical Microbiology 53:427-433. doi:10.1099/jmm.0.05472-0

Braat, H, Rottiers, P, Hommes, DW, Huyghebaert, N, Remaut, E, Remon, J, Van Deventer, SJ H, Neirynck, S, Peppelenbosch, MP, Steidler, L (2006) A phase I trial with transgenic bacteria expressing interleukin-10 in Crohn's disease. Clinical Gastroenterology and Hepatology 4:754-759. doi:10.1016/j.cgh.2006.03.028

Chopin, A, Chopin, MC, Moillo-Batt, A, Langella, P (1984) Two plasmid-determined restriction and modification systems in Streptococcus lactis. Plasmid 11:260-263. doi:10.1016/0147-619x(84)90033-7

Chung, CT, Niemela, SL, Miller, RH (1989) One-step preparation of competent Escherichia coli: transformation and storage of bacterial cells in the same solution. Proceedings of the National Academy of Sciences USA 86:2172-2175. doi:10.1073/pnas.86.7.2172

Cortez-Perez, NG, Poquet, I, Oliveira, M, Gratadoux, JJ, Madsen, SM, Miyoshi, A, Corthier, G, Azevedo, V, Langella, P, Bermúdez-Humarán, LG (2006) Construction and characterization of a Lactococcus lactis strain deficient in intracellular ClpP and extracellular HtrA proteases. Microbiology 152:2611-2618. doi:10.1099/mic.0.28698-0 
Cunningham, RP, Weiss, B (1985) Endonuclease III (nth) mutants of Escherichia coli. Proceedings of the National Academy of Sciences USA 82:474-478. doi:10.1073/pnas.82.2.474

Duarte, SOD (2018) Lactic acid bacteria as cell factories: a synthetic biology approach for plasmid DNA and recombinant protein production, Dissertation, Instituto Superior Técnico, University of Lisbon

Duarte SOD, Martins, MC, Andrade, SM, Prazeres, MF, Monteiro, GA (2019) Plasmid copy number of pTRKH3 in Lactococcus lactis is increased by modification of the repDE ribosome-binding site. Biotechnology Journal 14:1800587. doi:10.1093/nar/29.9.1975

Duarte, SOD, Monteiro, GA (2021) Plasmid replicons for the production of pharmaceutical-grade pDNA, proteins and antigens by Lactococcus lactis cell factories. International Journal of Molecular Sciences 22:1379. doi:10.3390/ijms22031379

Elliker, PR, Anderson, AW, Hannesson, G (1956) An agar culture medium for lactic acid streptococci and lactobacilli. Journal of Dairy Science 39:1611-1612. doi:10.3168/jds.S0022-0302(56)94896-2

Guo, T, Xin, Y, Zhang, Y, Gu, X, Kong, J (2019) A rapid and versatile tool for genomic engineering in Lactococcus lactis. Microbial Cell Factories 18:22. doi:10.1186/s12934-019-1075-3

Holo, H, Nes, IF (1989) High-frequency transformation, by electroporation, of Lactococcus lactis subsp. cremoris grown with glycine in osmotically stabilized media. Applied and Environmental Microbiology 55:3119-3123. doi:0099-2240/89/123119-05\$02.00/0

Huang, C, Ding, T, Wang, J, Wang, X, Guo, L, Wang, J, Zhu, L, Bi, C, Zhang, X, Ma, X, Huo, Y (2019) CRISPRCas9-assisted native end-joining editing offers a simple strategy for efficient genetic engineering in Escherichia coli. Applied Microbiology and Biotechnology 103:8497-8509. doi:10.1007/s00253-01910104-w

Huang, H, Zheng, G, Jiang, W, Hu, H, Lu, Y (2015) One-step high-efficiency CRISPR/Cas9-mediated genome editing in Streptomyces. Acta Biochimica et Biophysica Sinica 47:231-243. doi:10.1093/abbs/gmv007

Levy, MS, Lotfian, P, O’Kennedy, R, Lo-Yim, MY, Shamlou, PA (2000) Quantitation of supercoiled circular content in plasmid DNA solutions using a fluorescence-based method. Nucleic Acids Research 28:e57. doi:10.1093/nar/28.12.e57

Liu, F, Zhang, Y, Qiao, W, Zhu, D, Xu, H, Saris, PEJ, Qiao, M (2019) Restructured Lactococcus lactis strains with emergent properties constructed by a novel highly efficient screening system. Microbial Cell Factories 18:198. doi:10.1186/s12934-019-1249-z

Maguin, E, Duwat, P, Hege, T, Ehrlich, D, Gruss, A (1992) New themosensitive plasmid for Gram-positive bacteria. Journal of Bacteriology 174:5633-5638. doi:10.1128/jb.174.17.5633-5638.1992 
Maguin, E, Prévost, H, Ehrlich, SD, Gruss, A (1996) Efficient insertional mutagenesis in lactococci and other Gram-positive bacteria. Journal of Bacteriology 178:931-935. doi:10.1128/jb.178.3.931-935.1996

Matsumoto, Y, Zhang, Q, Takao, M, Yasui, A, Yonei, S (2001) Escherichia coli Nth and human hNTH1 DNA glycosylases are involved in removal of 8-oxoguanine from 8-oxoguanin/guanine mispairs in DNA. Nucleic Acids Research 29:1975-1981. doi:10.1093/nar/29.9.1975

Mierau, I, Kleerebezem, M (2005) 10 years of the nisin-controlled gene expression system (NICE) in Lactococcus lactis. Applied Microbiology and Biotechnology 68:705-717. doi:10.1007/s00253-005-01076

Miyoshi, A, Jamet, E, Commissaire, J, Renault, P, Langella, P, Azevedo, V (2004) A xylose-inducible expression system for Lactococcus lactis. FEMS Microbiology Letters 239:205-212. doi:10.1016/j.femsle.2004.08.018

Morello, E, Nouaille, S, Cortes-Perez, NG, Blugeon, S, Medina, LFC, Azevedo, V, Gratadoux, JJ, BermúdezHumarán, Le Loir, Y, Langella, $\mathrm{P}$ (2012) Inactivation of the $y b d D$ gene in Lactococcus lactis increases the amounts of exported proteins. Applied and Environmental Microbiology 78:7148-7151. doi:10.1128/AEM.01076-12

Muth, G, Nußbaumer, B, Wohlleben, W, Pühler, A (1989) A vector system with temperature-sensitive replication for gene disruption and mutational cloning in streptomycetes. Molecular and General Genetics 219:341-348. doi:10.1007/BF00259605

Muth, G, Wohlleben, W, Pühler, A (1988) The minimal replicon of the Streptomyces ghanaensis plasmid pSG5 identified by subcloning and Tn5 mutagenesis. Molecular and General Genetics 211:424-429. doi:10.1007/bf00425695

Nouaille, S, Commissaire, J, Gratadoux, JJ, Ravn, P, Bolotin, A, Gruss, A, Le Loir, Y, Langella, P (2004) Influence of lipoteichoic acid D-alanylation on protein secretion in Lactococcus lactis as revealed by random mutagenesis. Applied and Environmental Microbiology 70:1600-1607.

doi:10.1128/AEM.70.3.1600-1607.2004

O'Sullivan, DJ, Klaenhammer, TR (1993) High- and low-copy-number Lactococcusshuttle cloning vectors with features for clone screening. Gene 137:227-231. doi:10.1016/0378-1119(93)90011-Q

Pereira, VB, Saraiva, TDL, Souza, BM, Zurita-Turk, M, Azevedo, MSP, De Castro, CP, Mancha-Agresti, P, dos Santos, JSC, Santos, ACG, Faria, AMC, Leclereq, S, Azevedo, V, Miyoshi, A (2014) Development of a new DNA vaccine based on mycobacterial ESAT-6 antigen delivered by recombinant invasive Lactococcus lactis FnBPA+. Applied Microbiology and Biotechnology 99:1817-1826. doi:10.1007/s00253-014-6285-3

Pontes, DS, Dorella, FA, Ribeiro, LA, Miyoshi, A, Le Loir, Y, Gruss, A, Oliveira, SC, Langella, P, Azevedo, V (2003) Induction of partial protection in mice after oral administration of Lactococcus lactis producing 
Brucella abortus L7/L12 antigen. Journal of Drug Targeting 11:489-493. doi:10.1080/10611860410001670035

Saito, Y, Uraki, F, Nakajima, S, Asaeda, A, Ono, K, Kubo, K, Yamamoto, K (1997) Characterization of endonuclease III (nth) and endonuclease VIII (nei) mutants of Escherichia coli K-12. Journal of Bacteriology 179:3783-3785. doi:10.1128/jb.179.11.3783-3785.1997

Schneier, M, Razdan, S, Miller, AM, Briceno, ME, Barua, S (2020) Current technologies to endotoxin detection and removal of biopharmaceutical purification. Biotechnology and Bioengineering 117:25882609. doi:10.1002/bit.27362

Sinha, RP (1989) A new simple method of curing plasmids in lactic streptococci (Streptococcus cremoris; Streptococcus lactis, plasmids). FEMS Microbiology Letters 57:349-352. doi:10.1111/j.15746968.1989.tb03362.x

Song, X, Huang, H, Xiong, Z, Ai, L, Yang, S (2017) CRISPR-Cas9D10A nickase-assisted genome editing in Lactobacillus casei. Applied and Environmental Microbiology 83:e01259-17. doi:10.1128/AEM.01259-17.

van Mastrigt, O, Stefano, ED, Hartono, S, Abee, T, Smid, EJ (2018) Large plasmidome of dairy Lactococcus lactis subsp. lactis biovar diacetylactis FM03P encodes technological functions and appears highly unstable. BMC Genomics 19:620. doi:10.1186/s12864-018-5005-2

Vercoe, RB, Chang, JT, Dy, RL, Taylor, C, Gristwood, T, Clulow, JS, Richter, C, Przybilski, R, Pitman, AR, Fineran, PC (2013) Cytotoxic chromosomal targeting by CRISPR/Cas systems can reshape bacterial genomes and expel or remodel pathogenicity islands. PLoS Genetics 9:e1003454.

doi:10.1371/journal.pgen.1003454

Wakelin, SJ, Sabroe, I, Gregory, CD, Poxton, IR, Forsythe, JLR, Garden, OJ, Howie, SEM (2006) "Dirty little secrets" - Endotoxin contamination of recombinant proteins. Immunology Letters 106:1-7. doi:10.1016/j.imlet.2006.04.007

Wells, JM, Wilson, PW, Norton, PM, Gasson, MJ, Le Page, RWF (1993) Lactococcus lactis. high-level expression of tetanus toxin fragment $\mathrm{C}$ and protection against lethal challenge. Molecular Microbiology 8:1155-1162. doi:10.1111/j.1365-2958.1993.tb01660.x

Wicks, IP, Howell, ML, Hancock, T, Kohsaka, H, Olee, T, Carson, DA (1995) Bacterial lipopolysaccharide copurifies with plasmid DNA: Implications for animal models and human gene therapy. Human Gene Therapy 6:317-323. doi:10.1089/hum.1995.6.3-317

Zhu, D, Fu, Y, Liu, F, Xu, H, Saris, PEJ, Qiao, M (2017) Enhanced heterologous protein productivity by genome reduction in Lactococcus lactis NZ900. Microbial Cell Factories 16:1. doi:10.1186/s12934-0160616-2 
Zhu, D, Zhao, K, Xu, H, Zhang, X, Bai, Y, Saris, PEJ, Qiao, M (2014) Construction of thy A deficient Lactococcus lactis using the Cre-loxP recombination system. Annals of Microbiology 65:1659-1665. doi:10.1007/s13213-014-1005-x

\section{Figures}

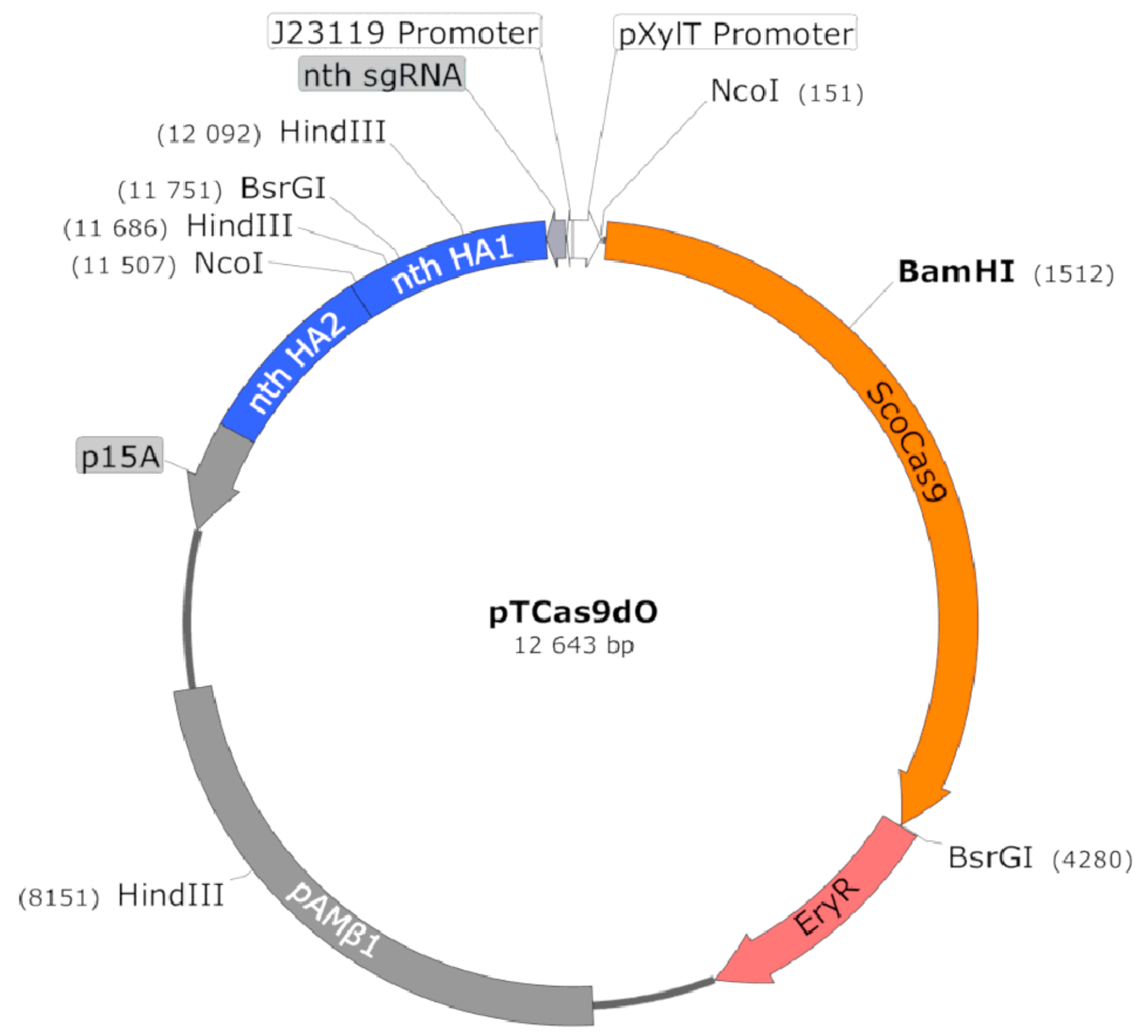

Figure 1

Schematics of the final constructed plasmids, pTCas9dO. Relevant restriction sites, for the BsrGI and Ncol (plasmid construction) and BamHI and HindIII (screening of transformants) enzymes were included. p15A - Gram-negative, medium copy number origin of replication; PAM 11 - Gram-positive, high copy 
number origin of replication; eryR - Erythromycin selection marker; pXylT Promoter - Xylose inducible PXyIT promoter; J23119 - Constitutive promoter; ScoCas9 - S. coelicor codon-optimized Cas 9 gene sequence; nth sgRNA - nth gene specific sgRNA; nth HA 1-2 - nth gene specific homology-arms or repair template, constituting the 1,028 (nth HA1) and 955 bp (nth HA2) fragments that share homology with the flanking regions of the nth gene; Image prepared using SnapGene software (from Insightful Science; available at snapgene.com).
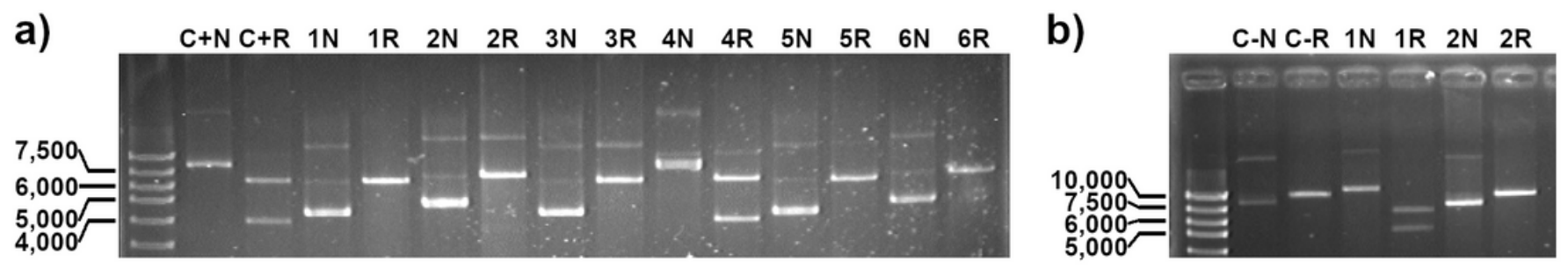

\section{Figure 2}

$1 \%(\mathrm{w} / \mathrm{V})$ agarose gels of every screening step of pTCas9dO construction. All molecular markers used were NZYDNA Ladder III (NZYTech). (a) Restriction profile of transformants obtained from pTScoCas9 ligation reaction using the BamHI and Hindlll enzymes. Non-restricted samples $(\mathrm{N})$ were ran alongside their restriction products (R). A positive control, composed of a previously obtained pTScoCas 9 solution unusable due to mutation ( $\mathrm{C}+$ ), was included. One clone (4) exhibited the correct pattern of 6,639 and $3,882 \mathrm{bp}$. (b) Restriction profile of transformants obtained from pTCas9dO ligation reaction using the BsrGI enzyme. A negative control (C-) was included using the pTScoCas9 plasmid. Non-restricted samples $(\mathrm{N})$ were ran alongside their restriction products $(\mathrm{R})$, and one clone (2) exhibited the correct restriction pattern of 7,471 and 5,172 bp.
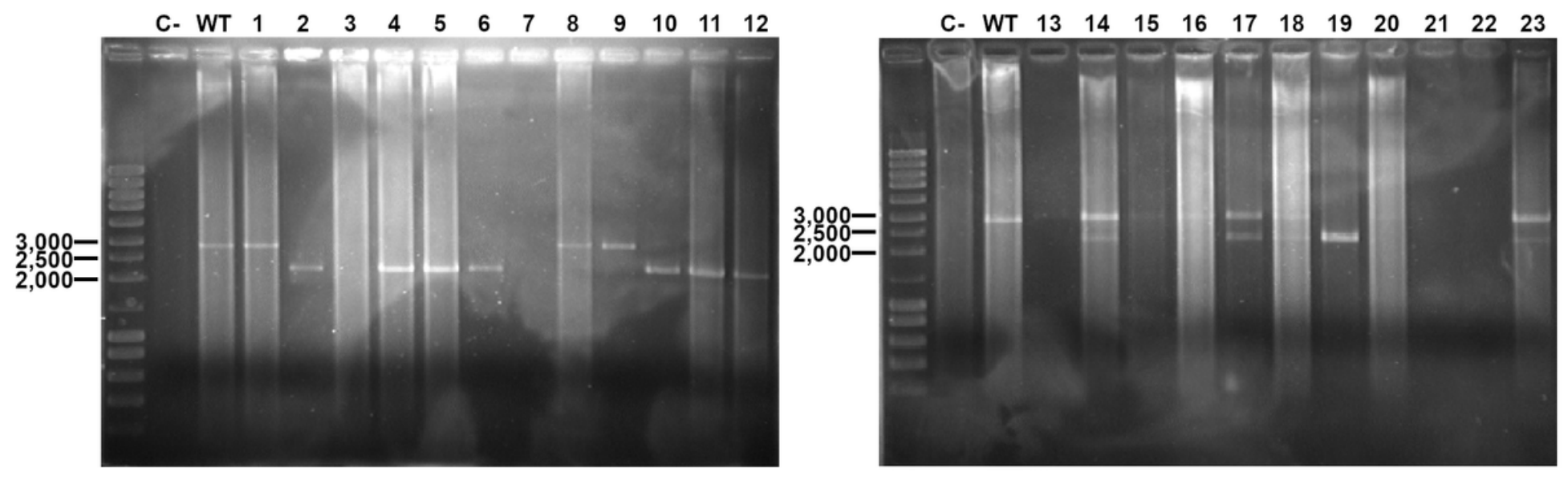

\section{Figure 3}

The pTCas9dO plasmid was transformed into L. lactis LMG19460 cells and the resulting transformants were induced by incubation in XM-17 medium. The induced cultures exhibited a noticeable decrease of cell density 3 hours after induction (OD $600 \mathrm{~nm}$ of $0.126 \pm 0.006$ to $0.083 \pm 0.006$ in 2 hours), most likely because of Cas9-induced cell death. The cultures maintained a lower cell density (OD600nm of roughly 
0.09) for over 24 hours following induction. Culture samples were plated after the cells were induced overnight. The resulting colonies were screened for the deletion by amplification of gDNA purified from individual colonies using the nth_conf_F/R primer pair (Figure 3).

a)

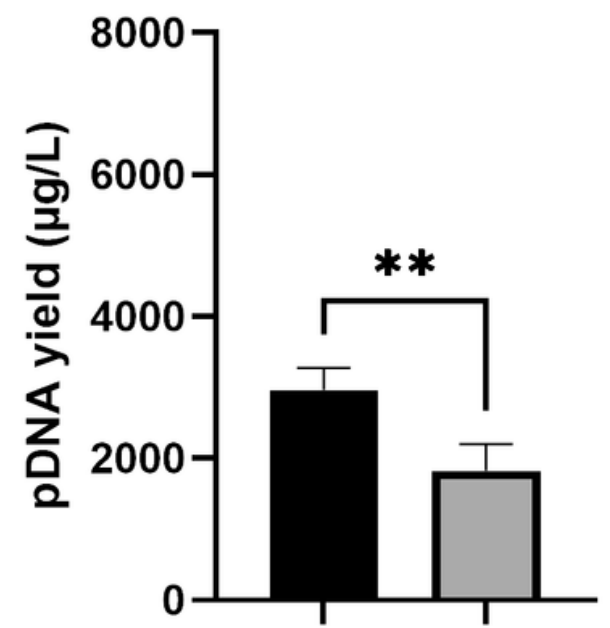

b)

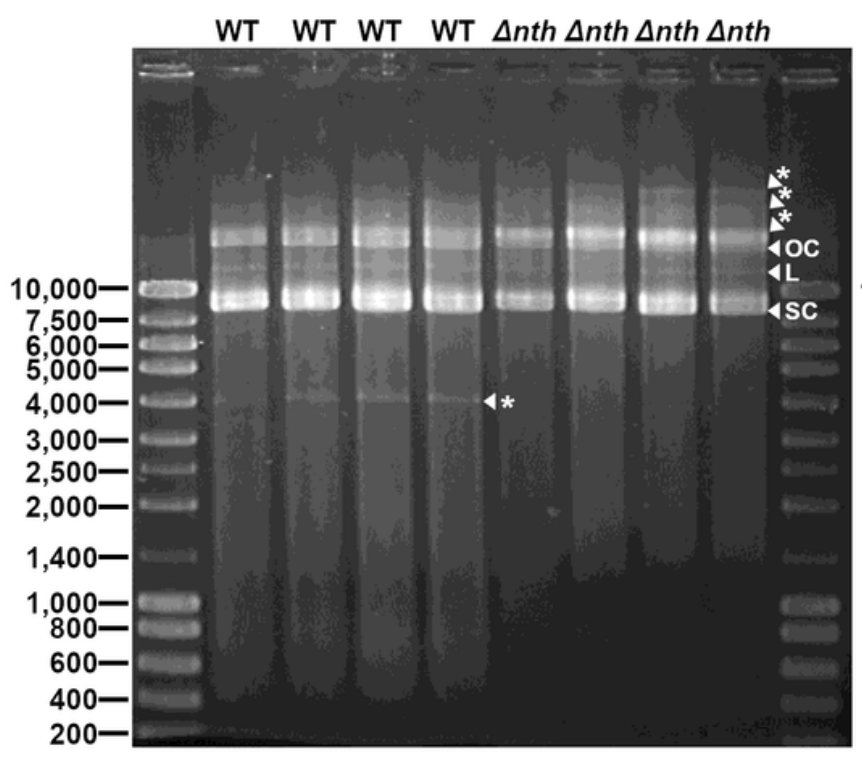

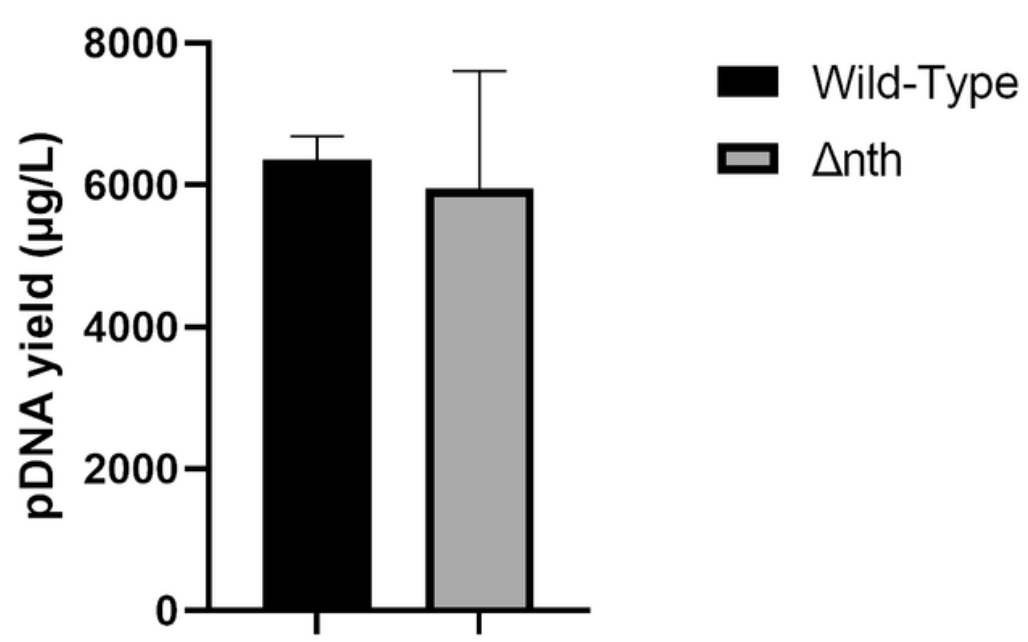

c)

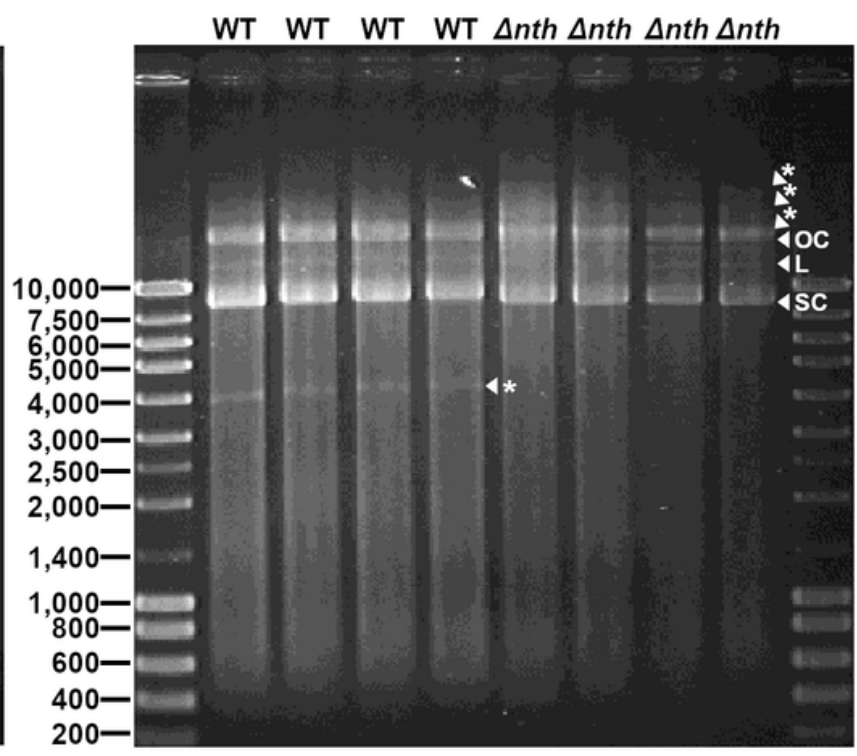

\section{Figure 4}

Results for the effect of the nth deletion on plasmid yield and quality. (a) Graph showing the average calculated plasmid yield ( $\mu \mathrm{g}$ per litre of culture), from duplicate cultures, for the wild-type and deletion mutant ( $\Delta$ nth) L. lactis LMG19460 cells, at late exponential (OD600nm of 1.6) and stationary (24 hours) phases of growth. The left graph shows results for the late-exponential phase while the right one shows results for the stationary phase. Asterisks represent statistically significant differences ( ${ }^{*}$ - P-value $\leq 0.05$, $\star \star$ - P-value $\leq 0.01)$ as determined by an unpaired two-tailed t-test. (b) $0.8 \%(\mathrm{w} / \mathrm{V})$ agarose gel of $2,000 \mathrm{ng}$ of the pTCas $9 \mathrm{dO}$ plasmid obtained from duplicate cultures of wild-type and $\Delta$ nth cells, at late-exponential (OD600nm of 1.6). (c) $0.8 \%(\mathrm{w} / \mathrm{V})$ agarose gel of 2,000 $\mathrm{ng}$ of the pTCas9dO plasmid obtained from duplicate cultures of wild-type and $\Delta$ nth cells and stationary (24 hours) phases of growth. The 
pTCas9dO's supercoiled (SC), open-circular (OC) and linear (L) isoforms are marked in each image. The unidentified bands are marked with an asterisk $\left(^{\star}\right)$. The molecular marker for every gel is the NZYDNA Ladder III (NZYTech).

\section{Supplementary Files}

This is a list of supplementary files associated with this preprint. Click to download.

- SupplementalFigure1.tif

- SupplementalFigure2.tif 